$\xi_{p}=-\mathrm{Z}$

\title{
Light speed expanding reversed black hole quantum cosmology
}

\author{
U. V. S. Seshavatharam ${ }^{1 *}$, S. Lakshminarayana ${ }^{2}$ \\ ${ }^{1}$ Honorary Faculty, I-SERVE, S. No-42, Hitex Road, Hitech City, Hyderabad-84, Telangana, India \\ ${ }^{2}$ Dept. of Nuclear Physics, Andhra University, Visakhapatnam-03, AP, India \\ *Corresponding author E-mail: seshavatharam.uvs@gmail.com
}

\begin{abstract} of light speed quantum cosmology.

\section{Introduction}

By considering 'Planck scale' as a characteristic limit of the evolving universe and by considering 'Black holes' as a deep cosmic probes, an evolving quantum cosmology with light speed expansion can be developed. Most interesting point to be noted is that, current cosmic physical parameters can be understood in a quantum gravitational approach.
\end{abstract}

With reference to ordinary matter, dark matter, critical density and time reversed black hole physics, it seems possible to develop a model

Keywords: Quantum cosmology; Black hole; Ordinary matter; Dark matter; Light speed expansion.

\section{Motivating points}

1) Now a days, cosmologists are seriously trying to explore the possibility of considering eternal light speed expansion of the universe [1].

2) So far nobody knows, why universe is allowing photon to travel at that much speed.

3) Light speed is a joint property of general theory of relativity and quantum mechanics and whole cosmological observations are based on photon. Like a photon, there is a scope for the universe to expand at speed of light.

4) Quantum gravity is a wide range physical model intended for understanding the in-built cosmological quantum phenomena on small scale as well as large scale distances. So far, progress in this direction is very nominal and general theory of relativity needs a serious review with reference to 'quantum cosmology'. In this context, Planck scale physics and black hole physics can be given some consideration [2-3].

5) When universe is giving birth to atoms, elementary parrticles and photons that show quantrum behaviour, universe can also be considered as a quantum gravitational object for ever.

6) When the early universe was able to create a number of primordial black holes, it may not be a big problem for the whole universe to behave like a primordial evolving black hole.

7) If it is assumed that, universe is a time reversed evolving black hole, there is a possibility of observing cosmic gravitational radiation.
8) With reference to particle physics, current technological limits on particle colliding energy, unidentified/unseen particles, unknown particle interactions and incomplete final unification scheme - to some extent, one can hopefully believe in the existence of dark matter.

9) Basically,'dark energy' was prposed for understanding cosmic acceleration. Careful analysis of improved supernovae data suggets that universe is coasting at constant veleocity and evidence for acceleration is only marginal [4,5]. In this context, now a days, a great debate has been initiated among mainstream copsmologists on the existence of dark energy [6] and needs further study. It is very crucial to note that, other than cosmic acceleration, so far cosmologists could not detect or address any single physical property of dark energy.

10) Eventhough redshift is an indication of cosmic expansion and galactic receding, without knowing the actual galactic distances and actual galactic receding speeds, with $100 \%$ confidence level, it may not be possible to decide the acelerating nature of cosmic expansion rate.

\section{Basic assumptions}

At any stage of cosmic evolution,

1) Total matter content is equal to the sum of ordinary matter content and dark matter content.

2) Critical density and total matter density are equal in magnitude.

3) Universe is a time reversed black hole with increasing Schwarzschild radius.

4) Following Hawking's black hole temperature formula, cosmic temperature is proportional to the square root of number of Planck masses.

Note-: There is no need to assume that, universe is expanding at light speed. It's an 'inbuilt' result of the first three assumptions. 


\section{General results}

$R_{t} \cong \frac{2 G M_{t}}{c^{2}}$

$M_{t} \cong \frac{c^{3}}{2 G H_{t}}$

$R_{t} H_{t} \cong c$

$T_{t} \cong \sqrt{\frac{M_{t}}{M_{p l}}}\left(\frac{\hbar c^{3}}{8 \pi G k_{B} M_{t}}\right)$

$\left.\cong \frac{\hbar c^{3}}{8 \pi G k_{B} \sqrt{M_{t} M_{p l}}} \cong \frac{\hbar \sqrt{H_{t} H_{p l}}}{4 \pi k_{B}}\right\}$

Age, $t \cong \frac{1}{H_{t}}$

Entropy, $S_{t} \cong \frac{\pi R_{t}^{2}}{L_{p l}^{2}} \cong 4 \pi\left(\frac{H_{p l}}{H_{t}}\right)^{2}$

where, $L_{p l} \cong \sqrt{\frac{G \hbar}{c^{3}}}$

\section{Adhoc relations for fitting current ordinary matter density and dark matter density}

We sincerely appeal that, for fitting the current values of ordinary and dark matter density ratios, we have proposed these relations. With further research, other such kind of relations can be developed using by which, in near future, trend of ordinary and dark matter density break up can be understood right from the beginning of Planck scale.

$$
\begin{aligned}
& \left(\Omega_{o m}\right)_{t} \cong\left(\frac{\beta_{t}}{1+\gamma_{t}}\right) \text { and }\left(\Omega_{d m}\right)_{t} \cong\left(\frac{\beta_{t}^{2}}{1+\gamma_{t}}\right) \\
& \left\{\left(\Omega_{o m}\right)_{t}+\left(\Omega_{d m}\right)_{t}\right\} \cong\left(\frac{\beta_{t}+\beta_{t}^{2}}{1+\gamma_{t}}\right) \text { and } \frac{\left(\Omega_{d m}\right)_{t}}{\left(\Omega_{o m}\right)_{t}} \cong \beta_{t} \\
& \text { where } \gamma_{t} \cong 1+\ln \left(\frac{H_{p t}}{H_{t}}\right) \text { and } \beta_{t} \cong\left(\frac{1+\sqrt{\gamma_{t}}}{2}\right)
\end{aligned}
$$

Based on relation (7), for the current case,

$$
\left.\begin{array}{l}
\left(\Omega_{o m}\right)_{0} \cong 0.0454,\left(\Omega_{d m}\right)_{0} \cong 0.29187 \text { and } \\
\left\{\left(\Omega_{o m}\right)_{0}+\left(\Omega_{d m}\right)_{0}\right\} \cong 0.33727 \\
\text { where } \gamma_{0} \cong 140.6024 \text { and } \beta_{0} \cong 6.4288
\end{array}\right]
$$

for the Planck scale,

$$
\left.\begin{array}{l}
\left(\Omega_{o m}\right)_{p l} \cong 0.5,\left(\Omega_{d m}\right)_{p l} \cong 0.5 \text { and } \\
\left\{\left(\Omega_{o m}\right)_{p l}+\left(\Omega_{d m}\right)_{p l}\right\} \cong 1.0 \\
\text { where } \gamma_{p l} \cong 1 \text { and } \beta_{p l} \cong 1
\end{array}\right]
$$

something else? Physicists expressed several opinions with many possible solutions. Most of the black hole physicists and cosmologists believe in the existence of primordial black holes. Probability of "universe being a priomordial evolving black hole" may be zero, but its possibility cannot be ruled out. In this context, in an optimistic approach, some of the modern cosmologists believe that, during the cosmic evolution, Planck scale quantum gravitational interactions might have an observable effect on the current observable cosmological phenomena. Clearly speaking, with respect to the current concepts of "Quantum gravity" and Planck scale early universal laboratory, current universe can be considered as a low energy scale laboratory. Thinking in this way, starting from the Planck scale, universe can be considered as a best quantum gravitational object. Based on relation (4), if recommended value [7] of $H_{0}$ is $(67.31 \pm 0.96) \mathrm{km} / \mathrm{sec} / \mathrm{Mpc}$, current estimated value of $T_{0}$ is $2.734 \mathrm{~K}$. With further reserch, ' $\Lambda$ term', 'inflation', 'horizon' and 'dark energy' like problems can be analysed in a quantum gravitational approach.

\section{Acknowledgements}

It is great pleasure to express our deep gratitude to Dr. E.Terry Tatum for his valuable technical discussions and kind encouragement. Author Seshavatharam is indebted to professors brahmashri M. Nagaphani Sarma, Chairman, shri K.V. Krishna Murthy, founder Chairman, Institute of Scientific Research in Vedas (I-SERVE), Hyderabad, India and Shri K.V.R.S. Murthy, former scientist IICT (CSIR), Govt. of India, Director, Research and Development, I-SERVE, for their valuable guidance and great support in developing this subject.

\section{References}

[1] Melia F., et al. 2016, the Epoch of Reionization in the $\mathrm{R}_{\mathrm{H}}=\mathrm{ct}$ Universe. Mon.Not.Roy.Astron.Soc. 456(4): 3422-3431. https://doi.org/10.1093/mnras/stv2902.

[2] Seshavatharam U. V. S and Lakshminarayana. S., 2016, On the Possible Role of Continuous Light Speed Expansion in Black Hole \& Gravastar Cosmology. Prespacetime Journal, Vol.7, Issue 3, 584-600.

[3] Tatum, E.T., Seshavatharam, U.V.S. and Lakshminarayana, S. 2015 the Basics of Flat Space Cosmology. International Journal of Astronomy and Astrophysics, 5: 116-124. https://doi.org/10.4236/ijaa.2015.52015.

[4] Neilsen J.T. et al., 2016, Marginal evidence for cosmic acceleration from Type Ia supernovae. Scientific Reports 6: 35596. https://doi.org/10.1038/srep35596.

[5] Lawrence H. Dam et al., 2017, Apparent cosmic acceleration due to large-scale peculiar motions. Mon.Not.Roy.Astron.Soc, 472 (2017) 835-851 https://doi.org/10.1093/mnras/stx1858.

[6] Balakrishna S. Haridasu et al. 2017, Strong evidence for an accelerating Universe. A\&A 600, L1

[7] Planck Collaboration: Planck 2015 Results. XIII. Cosmological Parameters.

\section{Discussion and conclusion}

Photons and black holes can be considered as the best candidates of quantum gravitational objects. It may not be wrong to say that, without the universe there is no independent existence to any photon or any black hole. Now the fundamental question to be answered is: Is the universe a quantum gravitational object or 